\title{
Endovascular thrombectomy with the AngioJet System for the treatment of intermediate-risk acute pulmonary embolism: a case report of two patients
}

\author{
Paweł Latacz ${ }^{1}$, Marian Simka², Tomasz Ludyga ${ }^{3}$, Tadeusz J. Popiela ${ }^{4}$, Tomasz Mrowiecki ${ }^{1}$ \\ ${ }^{1}$ Department of Vascular Surgery, University Hospital, Krakow, Poland \\ ${ }^{2}$ Department of Nursing, College of Applied Sciences, Ruda Śląska, Poland \\ ${ }^{3}$ Department of Vascular Surgery, EuroMedic, Katowice, Poland \\ ${ }^{4}$ Department of Radiology, Emergency and Mass-Event Medicine, Trauma Centre, University Hospital, Krakow, Poland
}

Adv Interv Cardiol 2016; 12, 1 (43): 61-64 DOI: $10.5114 /$ pwki.2016.56952

\section{Introduction}

Acute pulmonary embolism (PE) is one of the leading causes of death and ranks third, after myocardial infarction and cerebral stroke, among fatal hospital-related cardiovascular events [1-3]. Mortality associated with PE can be lowered by early treatments aimed at restoring patency of occluded pulmonary arteries (PAs). Although systemic thrombolysis remains the recommended management of PE [4], recent advances in endovascular techniques, especially of rheolytic thrombectomy, suggest that the endovascular approach can represent an alternative therapeutic strategy [5-9]. Here we describe endovascular treatment of two patients with acute PE. We used the AngioJet (Boston Scientific, Natick, MA, USA) rheolytic thrombectomy system. According to the available information, including personal communications, these were the second and third applications of this system in Poland for acute PE, and the first successful ones.

\section{Case reports}

Case 1. A 31-year-old man, without cardiovascular risk factors and no deep venous thrombosis on ultrasound examination, was admitted to our hospital due to a 3-day history of progressive dyspnea. There were electrocardiographic (ECG) features of overload of the right ventricle. Echocardiography demonstrated signs of right ventricle overload and increased pulmonary arterial pressure. Computed tomography (CT) angiography revealed saddle $\mathrm{PE}$, with partial occlusion of the pulmonary trunk, subtotal occlusion of the right PA and all its major branches, and partial occlusions of the branches of the left PA. Initially the patient received heparin, still with only a slight clinical improvement. Considering his severe clinical status, including profound hypoxemia, we decided to perform thrombectomy of the occluded PAs. The patient was consulted by a cardiovascular surgeon, who - taking into account the patient's clinical condition - instead of surgical thrombectomy recommended an endovascular approach.

Case 2. A 55-year-old woman, after chemo- and radiotherapy for small cell carcinoma of the right lung and clinical remission of the cancer, was transferred from the oncological department because of several-day history of increasing dyspnea. This patient developed PE despite adequate anticoagulation. Similarly to the previous case, there were ECG and echocardiographic signs of right ventricle overload and no sonographic features of deep venous thrombosis. CT angiography showed subtotal embolic occlusions of all major branches of the right PA and partial occlusions of branches of the left PA. Since this patient presented with lung cancer that represented a relative contraindication for open surgery of the chest, we also decided to manage PE endovascularly.

\section{Interventions}

As mentioned above, after a thorough diagnostic evaluation, including cardiovascular surgical consultations, in both cases we decided to address PE endovascularly. In both patients we used a similar technique. After cannulation of the femoral vein with a $5 \mathrm{Fr}$ introducer sheath, over the 0.035 -inch, $260 \mathrm{~cm}$ long hydrophilic guidewire (AqWire, Covidien, ev3 Endovascular, Inc., 

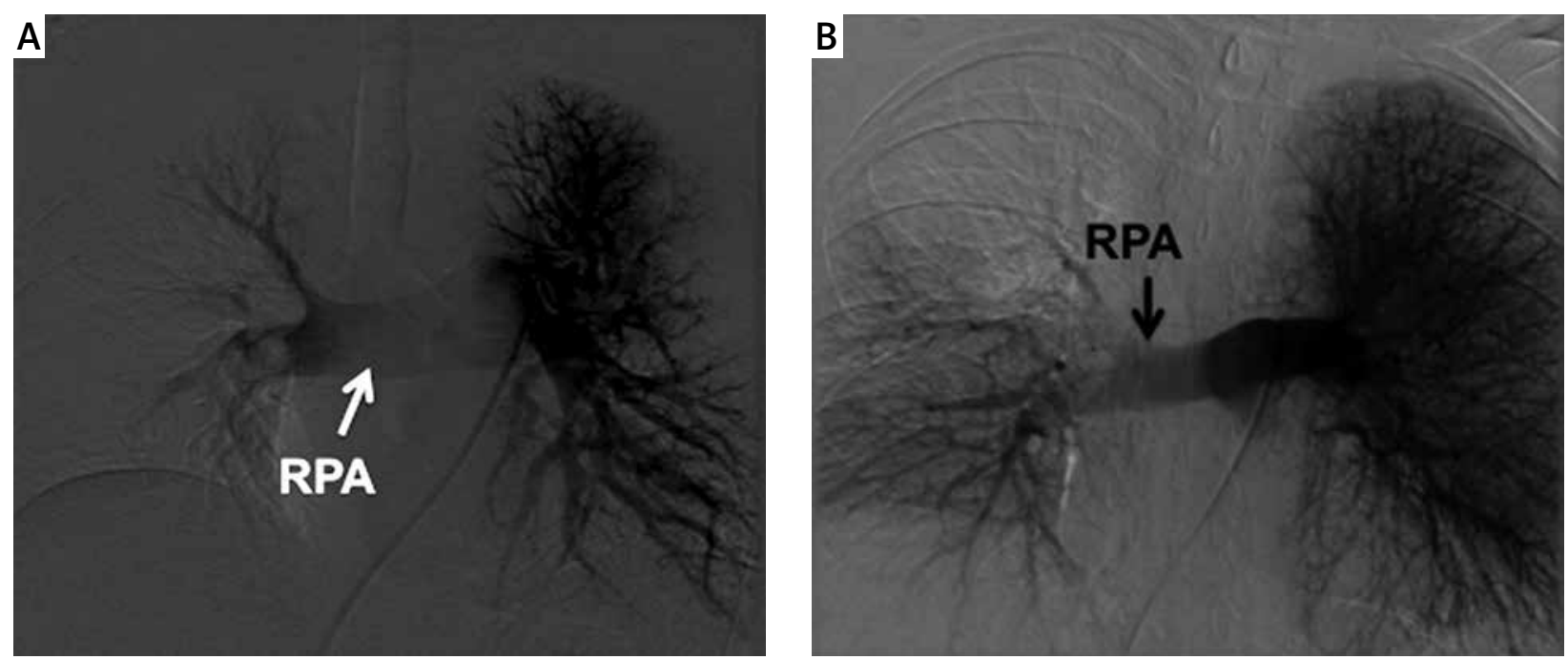

Figure 1. A - Initial angiography of the right PA in patient 1, B - initial angiography of the right PA in patient 2

RPA - Right pulmonary artery.

Plymouth, MN, USA), a Judkins Right diagnostic catheter was positioned in the pulmonary trunk. Then, the Judkins Right catheter was exchanged for a pig-tail one. Through this catheter we performed angiography of the PA. In the first patient angiography revealed embolic material in the middle branch of the left PA and massive embolism of all branches of the right PA (Figure $1 \mathrm{~A}$ ). In the other patient we found a small radiologic defect in the left PA and massive embolism of the branches of the right PA (Figure $1 \mathrm{~B}$ ). In both patients we injected $5000 \mathrm{U}$ of unfractionated heparin through the angiographic catheter. Then, we replaced the $5 \mathrm{Fr}$ introducer sheath with the $8 \mathrm{Fr}$ one, and navigated through the occlusion of the right PA with the 8 Fr Judkins Right catheter (Medtronic, Minneapolis, MN, USA), which was advanced to the distal part of the artery. Through this catheter, over a guidewire, we introduced the Angiolet system and performed thrombectomy, subsequently aspiring emboli from all occluded branches (Figures $2 \mathrm{~A}, \mathrm{~B}$ ). Firstly we removed emboli from the lower branch of the right PA, then we changed the position of the guidewire and AngioJet system and performed embolectomy of the middle and upper branches of the right PA. During aspirations both patients reported severe dyspnea followed by tachycardia, especially if the aspirations of embolic material lasted longer than 7-10 s. These clinical signs of circulatory decompensation lasted 6-8 s during initial aspirations, then they shortened to 2-4 s. Finally, in both cases we achieved good angiographic results (Figure $2 \mathrm{D}$ ) and the patients no longer demonstrated the above-mentioned signs of circulatory failure. Duration of the endovascular procedure in both cases was similar and was approximately 35-40 min.

The first patient, who was in a good clinical condition, was transferred to another hospital in order to perform more detail diagnostics, especially to find out the source of embolic material and to evaluate thoroughly his cardiovascular system. He was recommended to be administered rivaroxaban in a standard antithrombotic dose. The other patient 3 days after the endovascular procedure developed the symptoms of recurrent pulmonary thromboembolism. Computed tomography angiography confirmed embolism of the right PA. The patient received systemic thrombolysis with alteplase, which resulted in clinical improvement. Echocardiographic assessment, which was done 3 days later, revealed normal parameters of the right ventricle, with no signs of pulmonary hypertension. The patient was then transferred to the oncological department for further treatment of cancer.

\section{Discussion}

The main factor that can improve survival of patients with massive PE is early aggressive treatment aimed at restoring the patency of occluded PAs. According to guidelines of the European Society of Cardiology, systemic hypotension and shock represent accepted indications for urgent thrombolysis in patients with acute PE $[4,10]$. Still, although systemic intravenous thrombolysis in massive PE is considered a recommended and life-saving therapy, clinical benefit from this procedure is not fully confirmed [1]. According to data from the ICOPER registry, systemic thrombolysis does not significantly change mortality of patients with massive PE. In this study 90 -day mortality was $46.3 \%$ among patients receiving thrombolytic therapy and $55.1 \%$ in those without thrombolysis. Also recurrence of PE was not changed by thrombolysis and was $12 \%$ in both groups of patients [11]. The lack of clinical efficacy of systemic thrombolysis in massive PE is probably associated with difficulties obtaining patency of occluded PAs, since thrombolysis of 

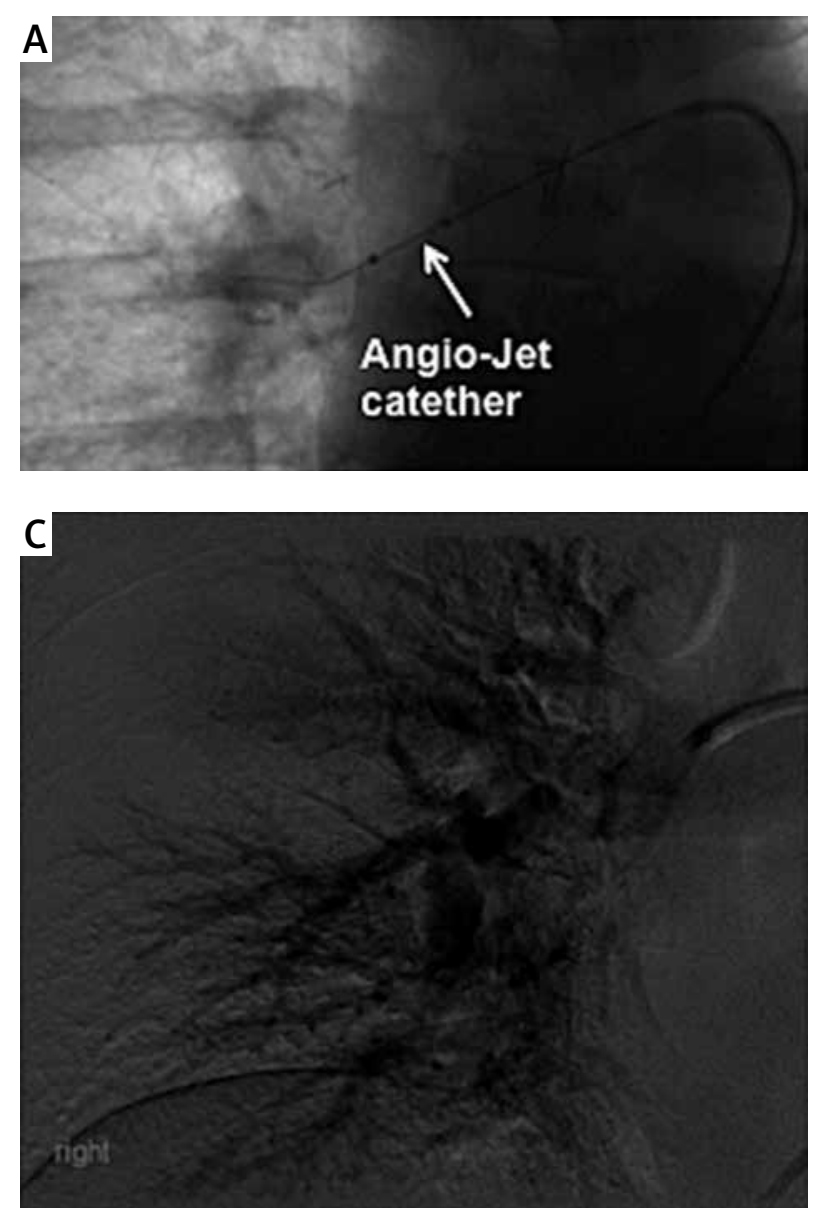

Figure 2. A, B - Passage of the catheter of the AngioJet system through the middle and upper branch of the right PA; C - Final result of the thrombectomy in patient 1 ; D - Final result of the thrombectomy in patient 2

partially occluded PAs is more effective [12]. Therefore, the guidelines recommend surgical embolectomy or catheter-directed treatment as an alternative to systemic thrombolysis for high-risk PE patients [4]. Administration of a thrombolytic agent directly into the lumen of the PA may accelerate lysis of the embolus and improve the rate of recanalization. For PE patients with a contraindication for surgical embolectomy and a contraindication for thrombolysis, endovascular interventions may consist of: fragmentation of the thrombus with a balloon catheter, rheolytic thrombectomy with hydrodynamic catheter devices (such as the system used in our patients), suction thrombectomy with aspiration catheters, or rotational thrombectomy. Endovascular techniques without fibrinolysis or using a reduced dose of fibrinolytic agent can be an alternative to standard thrombolytic treatment for PE [5-8, 13]. Still, current evidence supporting the use of these endovascular techniques is rather slim and more research is needed to unequivocally demonstrate their efficacy and safety.
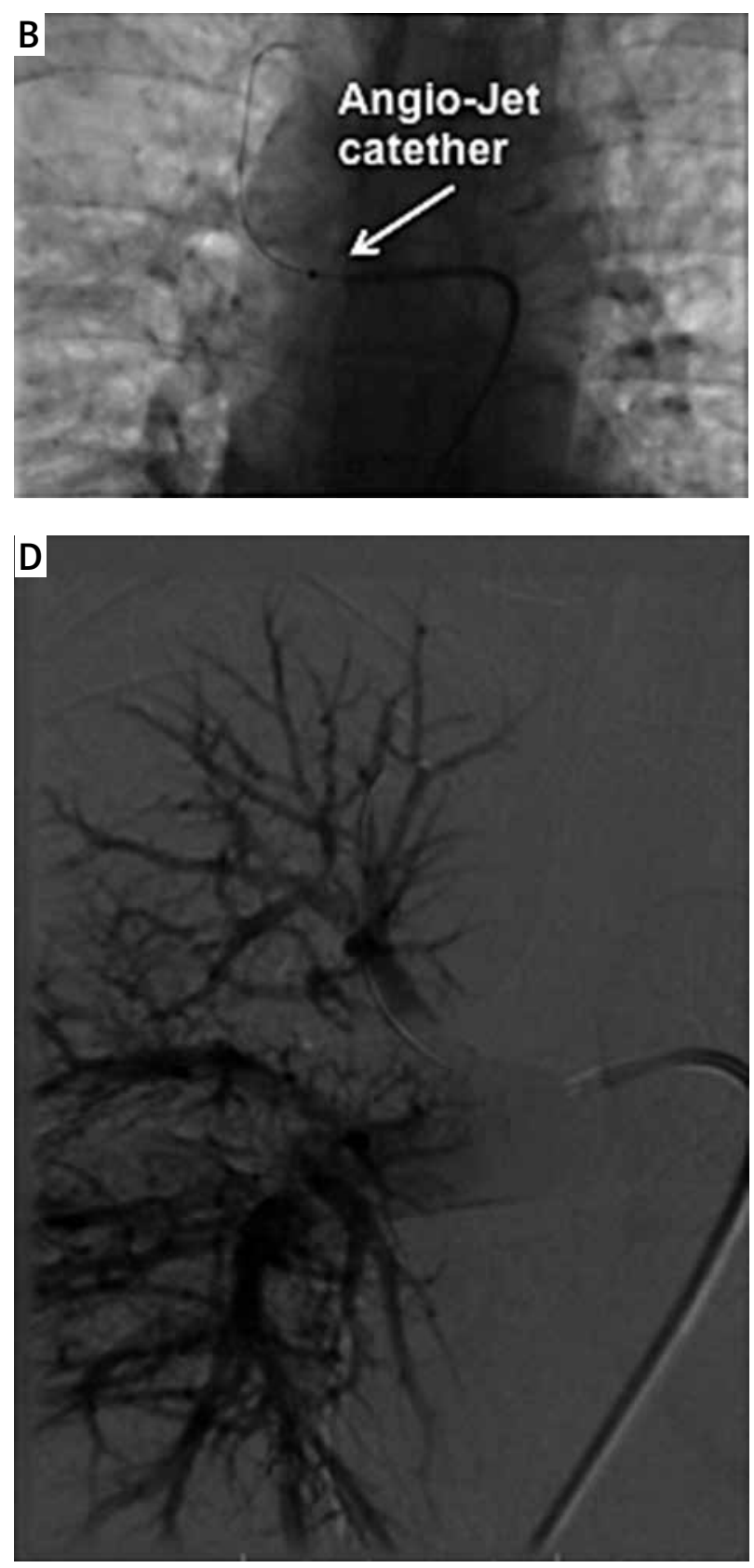

Case reports described in this paper confirm that in selected patients endovascular rheolytic thrombectomy can be a simple, safe and well-tolerated treatment modality for PE. We suggest that such a treatment should be used more often in Poland, primarily in PE patients with contraindications for other treatments, and also those who did not improve after standard anticoagulant and thrombolytic therapy and still demonstrate the symptoms of severe circulatory failure. However, such treatment should be performed in centers with good experience with this endovascular technique in other vascular territories and with full access to cardiovascular surgical intervention in case of a failed endovascular approach. 


\section{Conflict of interest}

The authors declare no conflict of interest.

\section{References}

1. Alikhan R, Peters F, Wilmott R, et al. Fatal pulmonary embolism in hospitalised patients: a necropsy review. J Clin Pathol 2004; 57: 1254-7.

2. Lindblad B, Eriksson A, Bergqvist D. Autopsy-verified pulmonary embolism in a surgical department: analysis of the period from 1951 to 1988. Br J Surg 1991; 78: 849-52.

3. Sandler DA, Martin JF. Autopsy proven pulmonary embolism in hospital patients: are we detecting enough deep vein thrombosis? J R Soc Med 1989; 82: 203-5.

4. Konstantinides SV, Torbiski A, Agnelli G, et al. 2014 ESC Guidelines on the diagnosis and management of acute pulmonary embolism. Eur Heart J 2014; 35: 3033-80.

5. Bunwaree S, Roffi M, Bonvini JM, et al. AngioJet ${ }^{\circledR}$ rheolytic thrombectomy: a new treatment option in cases of massive pulmonary embolism. Interv Cardiol 2013; 5: 71-87.

6. Margheri M, Vittori G, Vecchio S, et al. Early and long-term clinical results of Angiolet rheolytic thrombectomy in patients with acute pulmonary embolism. Am J Cardiol 2008; 101: 252-8.

7. Vecchio S, Varani E, Nuzzo A, et al. Percutaneous rheolityc thrombectomy with the Angio-Jet System for the treatment of intermediate-risk acute pulmonary embolism: a case report and an appraisal of contemporary indications and technique. Minerva Cardioangiol 2014; 62: 221-8.

8. Bonvini RF, Roffi M, Bounameaux $\mathrm{H}$, et al. AngioJet rheolytic thrombectomy in patients presenting with high-risk pulmonary embolism and cardiogenic shock: a feasibility pilot study. EuroIntervention 2013; 8: 1419-27.

9. De Gregorio MA, Gimeno MJ, Mainar A, et al. Mechanical and enzymatic thrombolysis for massive pulmonary embolism. J Vasc Interv Radiol 2002; 13: 163-9.

10. Pruszczyk P, Bochowicz A, Torbicki A, et al. Cardiac troponin T monitoring identifies high-risk group of normotensive patients with acute pulmonary embolism. Chest 2003; 123: 1947-52.

11. Kucher N, Rossi E, De Rosa M, et al. Massive pulmonary embolism. Circulation 2006; 113: 577-82.

12. Podbregar M, Krivec B, Voga G. Impact of morphologic characteristics of central pulmonary thromboemboli in massive pulmonary embolism. Chest 2002; 122: 973-9.

13. Latacz P, Rostoff P, Wyderka R, et al. Massive pulmonary embolism mimicking ST elevation acute coronary syndrome successfully treated with hybrid therapy in a trauma patient receiving nadroparin: diagnostic and therapeutic dilemmas. Kardiol Pol 2007; 65: 1235-42. 